\title{
Interactions of emotion and anxiety on visual working memory performance
}

\author{
Nick Berggren ${ }^{1}$ - Hannah M. Curtis ${ }^{1}$ - Nazanin Derakshan ${ }^{1}$
}

Published online: 15 December 2016

(C) Psychonomic Society, Inc. 2016

\begin{abstract}
It is a widely observed finding that emotion and anxiety interact; highly stressed or anxious individuals show robust attentional biases towards external negative information. More generally, research has suggested that exposure to threatening stimuli, as well as the experience of acute stress, also may impair top-down attentional control and working memory. In the current study, we investigated how the influence of emotion and anxiety may interact to influence working memory performance. Participants were required to encode the orientation of four simple shapes, eight, or four shapes while filtering out four other irrelevant shapes from memory. Before memory displays, an irrelevant neutral or fearful face cue also was presented. Memory performance was found to interact with self-reported state anxiety and cue valence; on neutral cue trials, state anxiety was negatively correlated with performance. This effect was absent following a fear cue. In addition, filtering efficiency was negatively associated with state anxiety solely following a fear cue. Our findings suggest that state anxiety's influence to visual working memory can be strongly modulated by external signals to threat. Most crucially, rather than anxious individuals having greater difficulty rejecting external threatening information, we observed that external threat may in its own right generally impair filtering efficiency in anxious individuals.
\end{abstract}

Nick Berggren

nbergg01@mail.bbk.ac.uk

1 Department of Psychological Sciences, Birkbeck University of London, Malet Street, WC1E 7HX London, UK
Keywords Anxiety · Emotion · Attention · Visual working memory $\cdot$ Individual differences $\cdot$ Cognition-emotion interactions

\section{Introduction}

Reciprocal links between emotion and attentional control have been well-documented in laboratory-based research. One hallmark finding from such research is that threat content appears to have a privileged role in attracting attention even when it is irrelevant to the task at hand (Yiend, 2010, for review). This effect is modulated by an individual's anxiety level; anxious individuals show heightened distractibility to threatening information in emotional Stroop and dot probe tasks (McNally, Kaspi, Riemann, \& Zeitlin, 1990; Bradley, Mogg, White, Groom, \& de Bono, 1999) and detect phobic-relevant stimuli at faster speeds (Öhman, Flykt, \& Esteves, 2001). This association between threat biases and anxiety has been demonstrated across anxiety disorders as well as within nonclinically anxious individuals (Cisler \& Koster, 2010, for review).

In addition to emotion and anxiety resulting in outcomes in cognition related to emotional stimuli, such as biased attention to threat, there are general effects on cognitive processes. Stress has a strong impact in impairing prefrontal cognitive abilities; prolonged stress even results in architectural changes in prefrontal dendrites (Arnstein, 2009, for review). For example, threat-induced anxiety disrupts spatial memory abilities (Shackman et al., 2006), and emotional distracting images similarly diminish prefrontal activity during memory tasks (Dolcos \& McCarthy, 2006). Meanwhile, trait anxiety generally disrupts aspects of attentional control, such as inhibition and shifting within standard selective attention tasks (Berggren \& Derakshan, 2013a, for review). 
Whereas there is ample support that threat and anxiety impair cognitive abilities, there also is surprising evidence that aspects of visual memory may be differentially influenced by these factors. Visual working memory is defined as the active short-term maintenance of information that is not currently perceptually available. Typically, this is assessed in change detection paradigms where participants are shown items to be remembered and, after a short delay period, see a new set of items and respond whether any items have changed. Rather than threat and anxiety impairing visual memory ability, there is evidence that encoding and maintaining threatening faces actually improves memory performance compared with neutral faces (Sessa, Luria, Gotler, Jolicœur, \& Dell'Acqua, 2011). In addition, self-reported anxiety also appears to correlate positively with memory performance for nonaffective items (e.g., bar orientations; Moriya \& Sugiura, 2012), although task-induced worry and state anxiety appear to have a contrary effect with reduced performance (Sari, Koster, \& Derakshan, in press). What is widely supported, however, is that when additional task-irrelevant information is added to displays that participants are told to ignore and not memorise, memory performance is impaired, with anxiety correlating with reduced filtering efficiency particularly when irrelevant information is threatening (Moriya \& Sugiura, 2012; Stout et al., 2013; Qi, Ding, \& Li, 2014).

Why might threat and anxiety, typically associated with impairments to cognitive processes, seemingly improve visual memory ability? One possibility is that, while visual working memory has traditionally been viewed as a late-stage cognitive process reflected by sustained maintenance activity within the prefrontal cortex (Sakai, Rowe, \& Passingham, 2002), there is growing evidence of sustained delay activity occurring within visual-perceptual areas and related to factors of spatial attention (Emrich, Riggall, LaRocque, \& Postle, 2013). This alternative "sensory recruitment" account (Awh \& Jonides, 2001; Postle, 2005) proposes that visual working memory maintenance primarily occurs in modality-specific sensory areas of the brain involved in perceptual analysis, whereas prefrontal activity may reflect higher-level control processes, such as inhibition of irrelevant information. Sustained spatial attention also appears to have a strong role in augmenting memory representations, with neural markers of delay/ maintenance period activity corresponding to the current focus of attention within working memory (Berggren \& Eimer, 2016; Lewis-Peacock, Drysdale, Oberauer, \& Postle, 2012). Following this account, there is evidence that both threat and anxiety may impact sensory processing in a beneficial fashion. For example, threat of electric shock increases peak amplitudes over early visual ERP components (e.g., the N1; Shackman, Maxwell, McMenamin, Greischar, \& Davidson, 2011), while viewing phobic-relevant images has similar impacts on early components, which contextualise to even include response to neutral images (Weymar, Keil, and Hamm,
2014; see also similar effects for internal negative affect: Schomberg, Schöne, Gruber, \& Quirin, 2016). Brief exposure to threatening faces also improves subsequent low-level contrast sensitivity (Phelps, Ling, \& Carrasco, 2006), appears to increase the processing of irrelevant visual information (Berggren \& Derakshan, 2013b), and associates with improved visual search efficiency under increasing demands on spatial attention (Becker, 2009). In addition, a recent study from our lab provided initial evidence that similar results can occur in individuals characterised by high anxiety, with greater detection sensitivity to small critical stimuli that appeared randomly while completing a visual search task of varying perceptual load (Berggren, Blonievsky, \& Derakshan, 2015).

Integrating this evidence, it is possible that the experience of threat, both from external or internal anxiety sources, may augment sensory intake as a means of promoting vigilance to threat in the visual environment (Eysenck, 1992), which has a counterintuitive benefit to visual working memory performance as this process strongly relies on sensory representation. Conversely, filtering efficiency of irrelevant information is impaired due to consequential increased sensory processing of irrelevant items, or an impairment in the ability to inhibit such items using topdown control. The present study explored this proposal in more detail and examined the role of both threat and anxiety in visual working memory performance simultaneously. Participants viewed threatening or neutral faces before the onset of a memory array. The brief exposure of threat versus nonaffect has been shown previously to elicit modulations to sensory processing (Phelps et al., 2006). During memory arrays, participants were instructed to encode and memorise the orientations of a varying number of red bars: four, eight, or four with an additional four blue bars interspersed among relevant items which participants were instructed to ignore. Following a delay period, participants were shown a test array where items either matched memory displays or differed, testing memory ability. We hypothesised that exposure to threat primes prior to memory arrays may transiently augment sensory information processing, resulting in improved perceptual encoding and subsequent visual memory performance compared with neutral primes (Sessa et al., 2011). In addition, we predicted that threat would impair performance in the additional irrelevant item condition where distracting items must be filtered out. Finally, we measured self-reported levels of trait and state anxiety prior to participants completing the task. Predictions regarding filtering efficiency performance were similar to that for external threat cues; anxiety should impair filtering efficiency based on previous evidence. For general working memory performance, we made no directional prediction as to whether anxiety would improve (Moriya \& Sugiura, 2012) or impair (Sari et al., in press) memory outcome based on conflicting evidence. 


\section{METHOD}

\section{Participants}

Fifty participants were recruited from Birkbeck University of London, via online advertisement (mean age $=27$ years, $\mathrm{SD}=$ 7; 12 males; 5 left-handed). Desired sample size was informed by assuming individual differences explaining at least $10 \%$ of variance similar to that of previous studies (i.e., correlation coefficient of .316), given an alpha level of 0.05 and statistical power of 0.8. Participants were given course credits for their participation. Data from eight participants were removed due to accuracy scores at chance-level within the most basic fouritem condition.

\section{Stimuli and Procedure}

E-Prime 2.0 (Psychology Software Tools, Inc.) was used to create and execute the experiment. Stimuli were presented on an 18-inch Mitsubishi Diamond Pro monitor $(60 \mathrm{~Hz} ; 1024 \mathrm{x}$ 768 screen resolution) at a viewing distance of approximately $60 \mathrm{~cm}$, controlled on a Dell Optiplex computer PC. Participants' responses were registered via keyboard button presses. Trial displays were presented on a black background with a grey fixation cross $\left(0.38^{\circ} \times 0.38^{\circ}\right.$ of visual angle $)$ that appeared throughout each experimental block. Face cue images $\left(2.86^{\circ} \times 4.77^{\circ}\right)$ presented during the task appeared at an eccentricity from fixation of $2.01^{\circ}$. There were eight facial identities used, four male and four female, and each displayed neutral or fearful expressions. Six identities were drawn from the NimStim set of facial expressions (Tottenham et al., 2009), and two from the Ekman Pictures of Facial Affect database (Ekman \& Friesen, 1976). Memory arrays consisted of four, eight, or four relevant with four irrelevant rectangles $\left(0.86^{\circ} \mathrm{x}\right.$ $\left.1.53^{\circ}\right)$. The number of rectangles each side of fixation was always even. Relevant rectangles appeared in red colour, and irrelevant rectangles in blue. All rectangles could appear in one of four possible orientations: vertical, horizontal, $45^{\circ} \mathrm{left}$, or $45^{\circ}$ right. Rectangles could appear on each side of fixation anywhere within an imaginary area measuring $5.25^{\circ}$ x $9.62^{\circ}$ at an eccentricity of $1.15^{\circ}$ from fixation. Stimuli were drawn from a large set of possible array images, including a 105 stimuli set for the four-item condition, 98 for the eight-item condition, and 101 for the four-item with distractors condition. Self-report trait and state anxiety level was measured prior to completion of the experiment using the State-Trait Anxiety Inventory (STAI; Spielberger, Gorsuch, Lushene, Vagg, \& Jacobs, 1983), a commonly used questionnaire to measure anxiety level with high internal consistency and test-retest reliability.

Each trial began with a fixation duration of $1500 \mathrm{~ms}$. A pair of faces (displaying a neutral or fearful expression) were then shown for $70 \mathrm{~ms}$, one both side of fixation, and both identical (see Fig. 1). Participants were instructed simply to ignore these images and maintain fixation during the trial. Following a short inter-stimulus interval of $30 \mathrm{~ms}$, the memory array appeared for $100 \mathrm{~ms}$. Participants were instructed to memorise the orientations of the red rectangles, and ignore any blue rectangles. A retention interval of $900 \mathrm{~ms}$ occurred, during which time the screen only contained the fixation cross. Finally, a test array appeared for $2000 \mathrm{~ms}$ or until response. Participants responded whether or not one of the red rectangles had changed in its orientation by pressing the " 2 " key for a change and the " 0 " key for no-change on the numeric keypad using their middle and index right fingers respectively. Participants' were given a 2,000-ms response window, after which time the trial was coded as incorrect. On trials containing additional irrelevant items, these also appeared in test arrays but never changed in their orientation. Following practice, participants completed six experimental blocks of 72 trials each. Each block contained six sets of trials, all presented in a random order within a block, with each set counterbalancing cue valence (2), change or no-change (2), and set size condition (3).

\section{Results}

Table 1 displays average accuracy scores across set size conditions, separately for the emotional valence of the prior face cue on trials. ${ }^{1}$ Accuracy was poorest in the set size 8 condition, though an initial $t$ test showed that this was significantly above chance-level $(t(41)=10.52, p<0.001)$. Accuracy in the set size 4 condition also seemed modulated by the presence of irrelevant items in the set size 4D condition.

\section{Working memory performance}

To assess influences of emotion, we conducted a $2 \times 2$ repeated-measures Analysis of Variance (ANOVA) ${ }^{2}$ on accuracy scores with the factors Cue Valence (Neutral, Fearful) and Set Size $(4,8)$. This showed a significant main effect of

\footnotetext{
${ }^{1}$ As our rationale was based on the notion of threat influencing sensory intake, and its proposed subsequent effect on change detection and visual working memory performance, we utilised memory accuracy as the outcome measure of performance rather than a theoretically-driven assumption of changes to an individual's quantitative capacity of visual memory (Pashler, 1988; for reference, general Pashler's $K$-value across set size was $M_{4}=2.34, M_{8}=1.69, M_{4 \mathrm{D}}$ $=1.85$ ). One benefit of capacity-based scores, however, is that they take into account the relationship between hit and false alarm rates of performance. All reported effects, however, unless otherwise stated, remained statistically significant at an alpha level of 0.05 when using d'prime sensitivity scores to control for this.

${ }^{2}$ We report separate analyses for the conditions $4 / 8$ and 4/4D based on distinction between basic visual memory performance and filtering efficiency, but note that an omnibus ANOVA containing all three condition levels still yielded a significant interaction by Cue Valence $(F(2,80)=3.23, p<0.05)$ to warrant such a decomposition.
} 


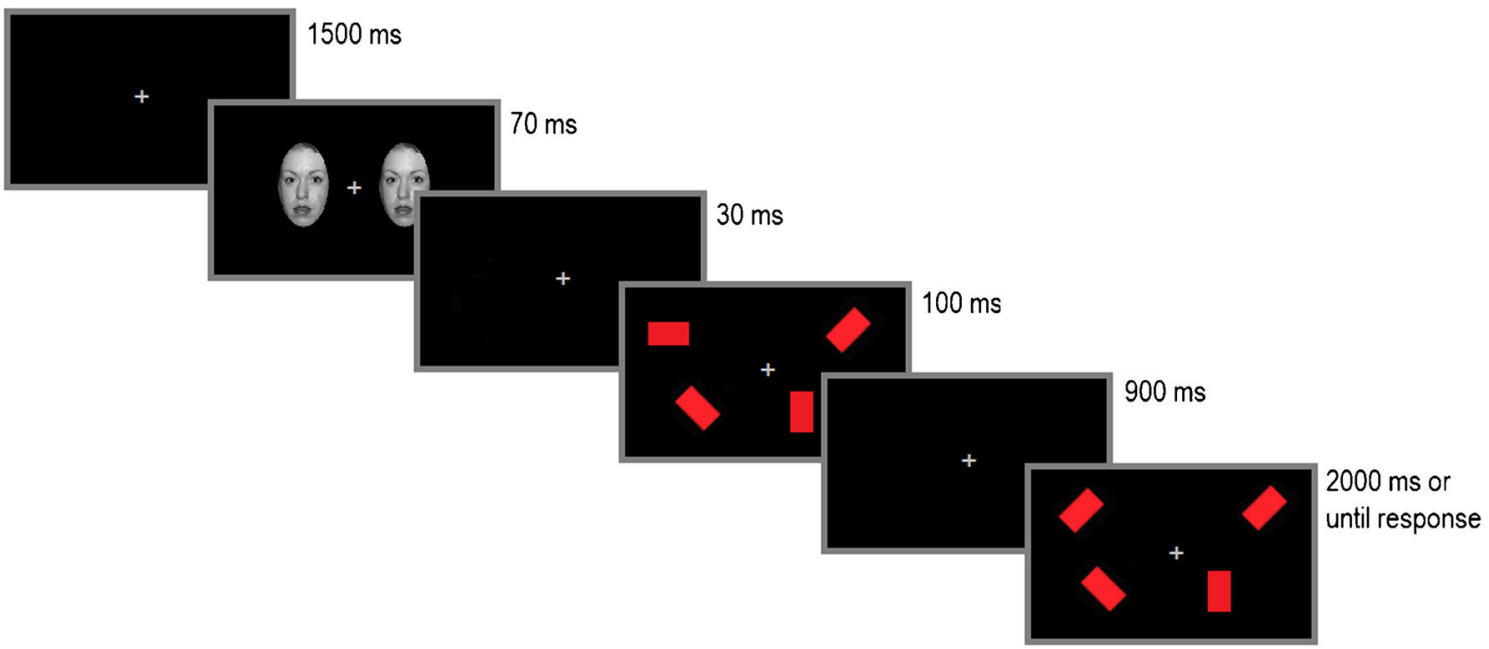

Fig. 1 Example experimental trial display (not to scale). Each trial contained two identical neutral (as shown) or fearful cues prior to memory arrays. Memory arrays contained four items to encode (as shown), eight, or four items with four irrelevant items (relevant items

Set Size $\left(F(1,41)=419.36, p<0.001, \eta_{\mathrm{p}}^{2}=0.91\right)$, showing that accuracy was higher under set size 4 versus $8(M=75 \%$ vs. $58 \%$ ). There was no significant main effect of Cue Valence or a two-way interaction $(F$ 's $<1)$.

Anxiety scores were then entered into Analysis of Covariance (ANCOVA) models separately for trait and state anxiety. Trait anxiety did not yield any significant main effect, two-interactions $(F$ 's $<1)$, or three-way interaction $(F(1,40)=$ $1.30, p>0.25)$. For state anxiety, there was no significant main effect $(F(1,40)=1.26, p>0.25)$, interaction with Set Size or three-way interaction $(F$ 's $<1)$. However, there was a significant interaction with Cue Valence $(F(1,40)=6.50, p<$ $\left.0.02, \eta_{\mathrm{p}}{ }^{2}=0.14\right)$. To decompose this, correlations were conducted assessing the relationship between state anxiety score and memory performance within the average of the two set size conditions, separately for each level of cue valence. Following neutral cues, state anxiety was negatively correlated with memory performance $(r=-0.311, N=42, p<0.05$; Fig. 2), whereas no correlation was seen following fear cues $(r$ $<0.10$ ). Subtracting accuracy on neutral trials from fear trials, performance change (i.e., a positive value indicating greater performance on fear trials) was positively correlated with state anxiety $(r=0.374, N=42, p<0.02$; Fig. 2$)$.

Table 1 Mean accuracy scores as a function of set size condition and cue valence (standard deviations in parentheses)

\begin{tabular}{|c|c|c|c|}
\hline & \multicolumn{3}{|c|}{ Set size } \\
\hline & 4 & 8 & $4 \mathrm{D}$ \\
\hline Neutral cue & $76(7)$ & $58(7)$ & $69(10)$ \\
\hline Fearful cue & $75(7)$ & $58(6)$ & $69(10)$ \\
\hline
\end{tabular}

were red, irrelevant items were blue). Following a maintenance delay period, participants were shown a test array and were asked to indicate whether the orientation of one of the relevant items had changed. Change and no-change trials were equally likely within blocks.

As an exploratory analysis, we examined whether this difference in performance may be due to anxiety associating with improved working memory performance following threat or whether lower levels of anxiety were associated with impaired performance following threat. To that end, we conducted a tertile split examining only high and low-level state anxious participants ( $\mathrm{N}=13$ vs. 12 , respectively; scores $<28$ and $>31$ ). Based on a significant interaction of Cue Valence $\mathrm{x}$ State Anxiety Group $\left(F(1,23)=9.17, p<0.01, \eta_{\mathrm{p}}{ }^{2}=0.29\right)$, we observed that low state anxious participants' performance significantly decreased following a fear cue $(M=67 \%$ vs. $71 \%$; $t(11)=2.75, p<0.02)$, with no difference for high state anxious participants $(M=65 \%$ vs. $68 \% ; t(12)=1.73, p>0.10)$. However, it should be noted that neither $t$ test remained statistically reliable when using more stringent d'prime sensitivity scores (both $p$ s $>0.05$ ).

\section{Filtering efficiency}

To examine filtering efficiency, a $2 \times 2$ repeated-measures ANOVA was conducted with the Set Size factor including set sizes 4 and 4D (i.e., without or with additional distractor items). Analysis showed a main effect of Set Size $(F(1,41)=$ $\left.39.01, p<0.001, \eta_{\mathrm{p}}{ }^{2}=0.49\right)$, in that the inclusion of distractor items significantly reduced memory performance for relevant items $(M=69 \%$ vs. $75 \%)$. There was no significant main effect of Cue Valence or interaction $(F$ 's $<1)$.

Conducting ANCOVA analyses for trait and state anxiety, trait anxiety score did not yield any main effect or two-way interactions $(F$ 's $<1)$, nor any three-way interaction with Set Size and Valence $(F(1,40)=1.99, p>0.15)$. For state anxiety score, there was a nonsignificant trend for a main effect of state anxiety $\left(F(1,40)=3.53, p=0.068, \eta_{\mathrm{p}}{ }^{2}=0.08\right)$ but a 


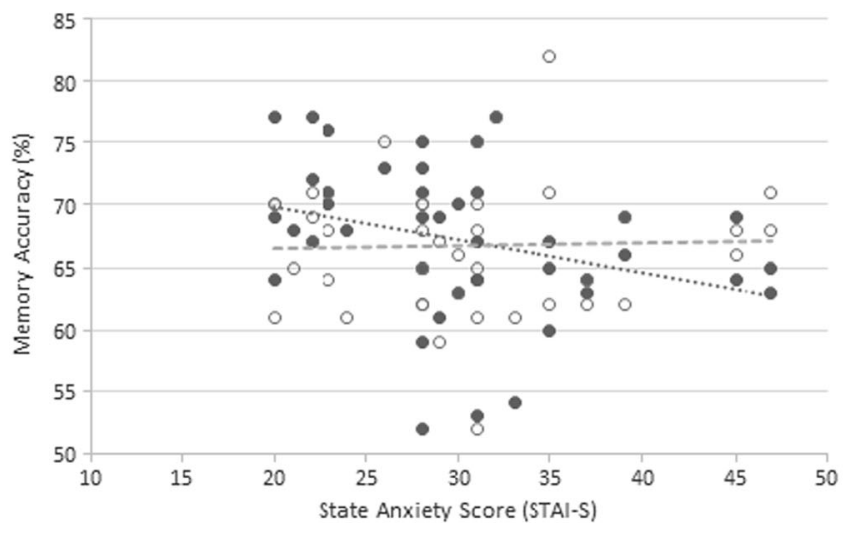

Fig. 2 Correlations between state anxiety score and memory performance (collapsed across set sizes 4 and 8 ). The left panel shows a negative correlation between state anxiety score and performance on neutral cue trials (represented by solid markers and a dotted trendline) along with, for comparison, performance on fear cue trials (unfilled

significant interaction with Set Size $(F(1,40)=6.67, p<0.02$, $\left.\eta_{\mathrm{p}}{ }^{2}=0.14\right)$, as well as a significant three-way interaction with Set Size and Cue Valence $\left(F(1,40)=5.03, p<0.05, \eta_{\mathrm{p}}{ }^{2}=\right.$ $0.11)$. Decomposing this, following a neutral cue, state anxiety score did not elicit a significant main effect on performance $(F(1,40)=3.44, p=0.07)$ and did not interact with Set Size $(F$ $<1)$. Following a fearful cue, again there was no main effect of state anxiety $(F(1,40)=2.57, p>0.10)$, but scores interacted with Set Size $\left(F(1,40)=12.36, p=0.001, \eta_{\mathrm{p}}^{2}=.24\right)$. Correlations showed that state anxiety did not influence performance in the set size 4 condition $(r<0.1)$ but was negatively correlated with accuracy in the 4D condition $(r=$ $-0.388, N=42, p=0.01$ ).

To further scrutinise these results, we calculated filtering efficiency scores as a subtraction of accuracy scores in the set size 4D condition from those in the set size 4 condition (i.e., "4" minus "4D"; more positive values indicate poorer filtering). General filtering efficiency scores correlated positively with state anxiety $(r=0.416, N=42, p<0.01)$. However, this effect was not present when examining neutral cue trials $(r=$ $0.164 N=42, p>0.20)$ and occurred solely on fear cue trials $(r$ $=0.508, N=42, p=0.001$; Fig. 3 ).

\section{Discussion}

The present study examined the role of internal (individual levels of anxiety) and external (emotional face cues) emotion in visual working memory performance. Results showed a number of interactions between these factors. First, state anxiety was associated with impaired accuracy following a neutral cue, with this effect not present following threat. In addition, filtering efficiency was negatively correlated with state anxiety but was specifically the case following a threat cue.

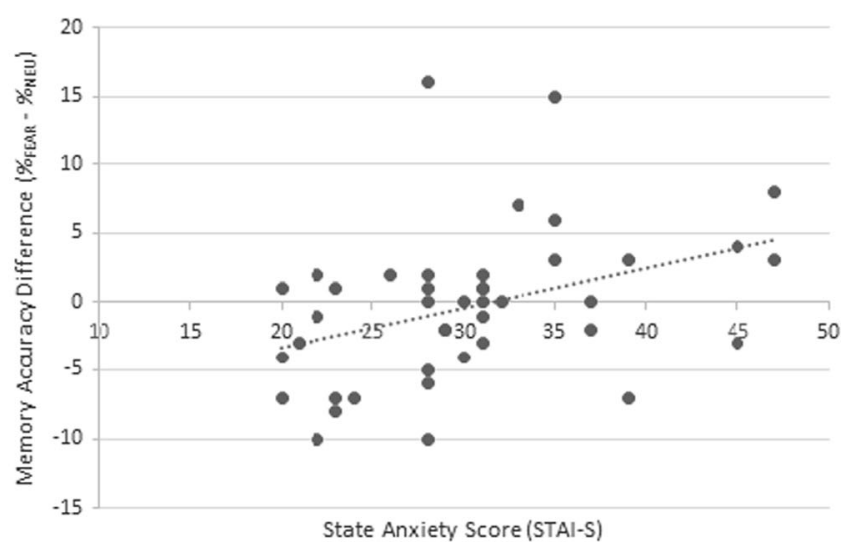

markers and a striped trendline). The right panel demonstrates a positive correlation between state anxiety score and a derived performance score based on accuracy on fearful cue trials minus neutral cue trials (i.e., more negative values indicating worse memory performance on fear trials).

\section{Visual memory performance}

The finding that state anxiety was associated with poorer performance following neutral face cues is consistent with Sari et al. (in press), who reported matching effects following the induction of state worry and anxiety on basic visual working memory performance. However, they discord from other evidence that anxiety may generally improve visual memory in the absence of accompanying task-irrelevant stimuli (Moriya \& Sugiura, 2012). It is important to note that those effects occurred within high trait anxious individuals characterised by social anxiety. In the present study, we found no evidence of a modulation to memory performance in either direction by trait anxiety. It therefore remains possible that trait anxiety may have an opposing influence in improving visual memory performance. Nevertheless, considering that high trait anxious individuals typically also report high state anxiety (in the present study, $r=0.594, N=42, p<0.001$ ), it is unclear how trait anxiety might link with improved visual memory while acute

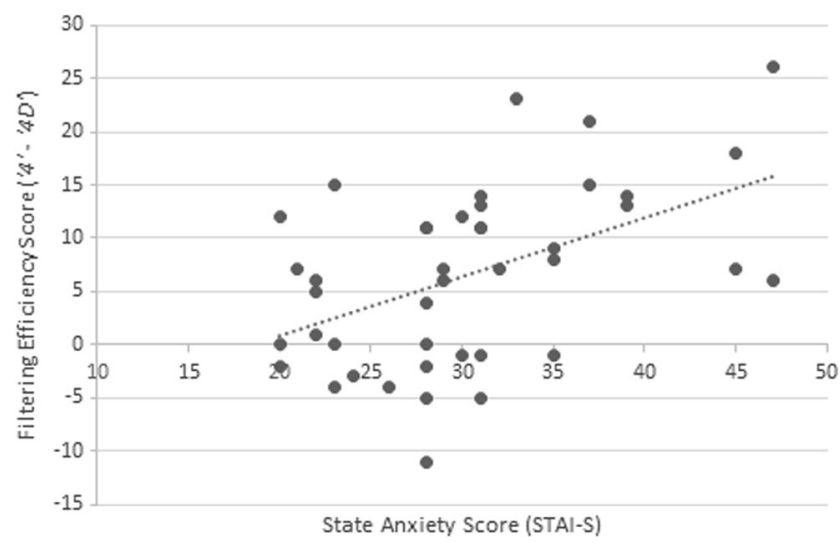

Fig. 3 Positive correlation between state anxiety score and filtering efficiency score for fear cue trials. Larger positive values indicate poorer filtering efficiency. 
anxiety simultaneously corresponds with impairment of this process, as observed in the present study. Future research should further investigate the proposed beneficial role of trait anxiety in visual working memory before contrasting this with more direct mood induction procedures of state anxiety.

While state anxiety was associated with impaired general performance following a neutral face cue, this effect was not evident following a threat cue. Due to the nature of this interaction based on a continuous variable, it is difficult to interpret whether this change in performance could relate to higher anxious individuals improving in performance on fear cue trials, or due to lower anxious individuals' performance more strongly deteriorating on fear cue trials. In our exploratory analysis using a tertile split to create high and low anxiety groups, we found some evidence to suggest that low anxious individuals' performance was specifically impaired following a fear cue. While our findings tentatively point to threat primarily impairing performance in low state anxious individuals, resulting in a synonymous effect to performance as seen in internalised high state anxiety following neutral cues, this possibility requires direct investigation in future research.

Indeed, even if external threat cues predominantly impaired visual memory performance (in the case of low anxious individuals), this also discords from evidence that threat signals generally should improve early information processing and sensory acquisition (Phelps et al., 2006), which we hypothesised might translate to greater visual memory performance as when emotional information itself is maintained (Sessa et al., 2011). This can be reconciled within the present study, if one assumes that the influence of external threat on sensory processing does not extend beyond initial perception to the level of visual memory performance. Indeed, whereas Sessa et al. (2011) found strong evidence of improved maintenance, this may be due to the emotional information itself being memorised. In our study, emotion was incidental to the task, and so external threat exposure may only beneficially impact visual working memory when emotional material itself is maintained.

\section{Filtering efficiency}

State anxiety was associated with impaired filtering following a fear cue. Surprisingly, considering poorer general performance after neutral cues, filtering was not modulated for this condition. The observation of impaired filtering in anxious individuals in relation to threat, however, is consistent with findings by Stout and colleagues $(2013 ; 2015)$ where filtering efficiency was not compromised for neutral items. What is particularly notable within the present data is that fear cues were presented prior to memory arrays and were not relevant items to be memorised themselves. Because of this, our data suggest that, rather than anxious individuals having difficulty filtering out threatening information (Stout et al., 2013), threat may in itself impair the prioritisation of relevant over irrelevant signals within visual memory for acutely anxious individuals, acting separately from the general effect of internal/ external threat on basic memory performance.

This finding may have broader implications; previous investigations often have argued the effect of anxiety on attentional biases to threat, but there is debate regarding whether such biases are due to a) anxious individuals being more prone to attentional capture by threat, b) all individuals being captured but anxious individuals showing a delayed ability to disengage attention from threat (Steppes, Luria, Fukuda, \& Gross, 2013), or c) a combination of the two (Fox, Russo, \& Dutton, 2002; see also Fukuda \& Vogel, 2009, 2011, for similar debate regarding enhanced distraction in individuals with low working memory capacity). On the basis of the present study, delayed disengagement evidence could be explained by the assumption that threat causes impaired selective attentional processes in anxious individuals. A key avenue of future research would be to assess how exposure to threat may influence inhibitory processes within standard selective attention tasks as opposed to visual memory experiments. Based on the present study, one would predict that interference by task-irrelevant information may increase in anxiety following threat exposure, implying a more broad disruption to attentional control processes in anxiety after witnessing a threat-related event (see Kalanthroff, Henik, Derakshan, \& Usher, 2016, for initial evidence in Stroop task performance).

We have interpreted results in that basic visual memory performance is impaired by threat, which can clearly be defined internally (i.e., the role of stress and acute anxiety) and, to some extent, also may be defined as externally-driven (i.e., with some preliminary evidence of similar effects through presentation of threat-relevant stimuli in lower anxious individuals). In contrast, the effect of threat on filtering efficiency might require the interaction of internal and external threat, as state anxiety on its own did not influence filtering on neutral trials, and nor was there any evidence of an association of poorer performance for low state anxious individuals following fear cues. However, it is fair to argue that contextualised factors of emotion could have influenced performance, which might compel a tempering of this conclusion. For instance, when threat stimuli were included in previous studies, effects on memory performance and filtering in relation to anxiety were exclusive to these stimuli (Stout et al., 2013; 2015) but, in tasks with solely neutral stimuli, the same results for these items also have been reported (Qi et al., 2014; Sari et al., in press; Stout \& Rokke, 2010). Thus, the lack of an influence of anxiety on filtering efficiency following neutral cues in the present study does not entirely preclude the idea that internalised anxiety is capable of disrupting filtering efficiency in its own right. Indeed, this interpretation would also better conform to the body of evidence that anxiety can disrupt 
inhibitory processes in a variety of selective attention tasks (Berggren \& Derakshan, 2013a). Alternatively, the choice of presenting cues before encoding may have influenced the precise nature of interactions with state anxiety. Previous studies examining the role of threat and anxiety in modulating filtering efficiency performance have varied where threatening information is presented within trials, such as at encoding (Stout et al., 2013) or during the delay period where information is being maintained (Dolcos \& McCarthy, 2006). There is evidence to suggest that such a choice is not arbitrary; McNab and Dolan (2014) found that distractors presented at encoding and during maintenance each account for unique variance in visual memory performance. Because our emotional cues were presented before encoding, we assume that our effects on filtering efficiency are akin to the influences of threat at encoding, but it is possible that our results also reflect a different mechanism. Future research could benefit from examining potential differences in anxious filtering efficiency when threat is presented as a prior cue, at encoding, or during delay period maintenance.

We conclude that the experience of internalised stress and anxiety is associated with impaired visual working memory performance. As an independent factor, filtering efficiency also may be impaired by purely internally driven anxieties, but conversely is shown here to be strongly impacted by the interaction of internal anxiety and the presentation of an external threat in the visual environment. Our findings build on previous research highlighting attentional biases to threat in anxiety, showing that external threat may in its own right disrupt filtering efficiency processes when experiencing high anxiety. These results may assist in elucidating the underlying mechanisms behind impaired attentional control seen in anxious populations.

\section{References}

Arnstein, A. F. (2009). Stress signalling pathways that impair prefrontal cortex and function. Nature Reviews Neuroscience, 10, 410-422.

Awh, E., \& Jonides, J. (2001). Overlapping mechanisms of attention and spatial working memory. Trends in Cognitive Sciences, 5, 119-126.

Becker, M. W. (2009). Panic search: Fear produces efficient visual search for nonthreatening objects. Psychological Science, 20, 435-437.

Berggren, N., Blonievsky, T., \& Derakshan, N. (2015). Enhanced visual detection in trait anxiety. Emotion, 15, 477-483.

Berggren, N., \& Derakshan, N. (2013a). Attentional control deficits in trait anxiety: Why you see them and why you don't. Biological Psychology, 92, 440-446.

Berggren, N., \& Derakshan, N. (2013b). Blinded by fear? Prior exposure to fearful faces enhances attentional processing of task-irrelevant stimuli. Quarterly Journal of Experimental Psychology, 66, 22042218.

Berggren, N., \& Eimer, M. (2016). Does contralateral delay activity reflect working memory storage or the current focus of spatial attention within visual working memory? Journal of Cognitive Neuroscience, 28, 2003-2020.

Bradley, B. P., Mogg, K., White, J., Groom, C., \& de Bono, J. (1999). Attentional bias for emotional faces in generalised anxiety disorder. British Journal of Clinical Psychology, 38, 267-278.

Cisler, J. M., \& Koster, E. H. (2010). Mechanisms of attentional biases towards threat in anxiety disorders: An integrative review. Clinical Psychology Review, 30, 203-216.

Dolcos, F., \& McCarthy, G. (2006). Brain systems mediating cognitive interference by emotional distraction. Journal of Neuroscience, 26, 2072-2079.

Ekman, P., \& Friesen, W. V. (1976). Pictures of facial affect. Palo Alto, CA: Consulting Psychologists Press.

Emrich, S. M., Riggall, A. C., LaRocque, J. J., \& Postle, B. R. (2013). Distributed patterns of activity in sensory cortex reflect the precision of multiple items maintained in visual short-term memory. Journal of Neuroscience, 33, 6516-6523.

Eysenck, M. W. (1992). Anxiety: The cognitive perspective. Hove, England: Erlbaum.

Fox, E., Russo, R., \& Dutton, K. (2002). Attentional bias for threat: Evidence for delayed disengagement from emotional faces. Cognition and Emotion, 16, 355-379.

Fukuda, K., \& Vogel, E. K. (2009). Human variation in overriding attentional capture. The Journal of Neuroscience, 29, 8726-8733.

Fukuda, K., \& Vogel, E. K. (2011). Individual differences in recovery time from attentional capture. Psychological Science, 22, 361-368.

Kalanthroff, E., Henik, A., Derakshan, N., \& Usher, M. (2016). Anxiety, emotional distraction, and attentional control in the Stroop task. Emotion, 16, 293-300.

Lewis-Peacock, J. A., Drysdale, A. T., Oberauer, K., \& Postle, B. R. (2012). Neural evidence for a distinction between short-term memory and the focus of attention. Journal of Cognitive Neuroscience, 24, 61-79.

McNab, F., \& Dolan, R. J. (2014). Dissociating distractor-filtering at encoding and during maintenance. Journal of Experimental Psychology: Human Perception and Performance, 40, 960-967.

McNally, R. J., Kaspi, S. P., Riemann, B. C., \& Zeitlin, S. B. (1990). Selective processing of threat cues in posttraumatic stress disorder. Journal of Abnormal Psychology, 99, 398-402.

Moriya, J., \& Sugiura, Y. (2012). High visual working memory capacity in trait social anxiety. PLoS One, 7, e34244.

Öhman, A., Flykt, A., \& Esteves, F. (2001). Emotion drives attention: Detecting the snake in the grass. Journal of Experimental Psychology: General, 130, 466-478.

Pashler, H. (1988). Familiarity and visual change detection. Perception \& Psychophysics, 44, 369-378.

Phelps, E. A., Ling, S., \& Carrasco, M. (2006). Emotion facilitates perception and potentiates the perceptual benefits of attention. Psychological Science, 17, 292-299.

Postle, B. R. (2005). Delay-period activity in the prefrontal cortex: One function is sensory gating. Journal of Cognitive Neuroscience, 17, 1679-1690.

Qi, S., Ding, C., \& Li, H. (2014). Neural correlates of inefficient filtering of emotionally neutral distractors from working memory in trait anxiety. Cognitive, Affective, \& Behavioral Neuroscience, 14, 253-265.

Sakai, K., Rowe, J. B., \& Passingham, R. E. (2002). Active maintenance in prefrontal area 46 creates distractor-resistant memory. Nature Neuroscience, 5, 479-484.

Sari, B.A., Koster, E.H., \& Derakshan, N. (in press). The effects of active worrying on working memory capacity. Cognition and Emotion.

Schomberg, J., Schöne, B., Gruber, T., \& Quirin, M. (2016). Emotion and hypervigilance: Negative affect predicts increased P1 responses to non-negative pictorial stimuli. Experimental Brain Research, 234, $1395-1402$. 
Sessa, P., Luria, R., Gotler, A., Jolicœur, P., \& Dell'Acqua, R. (2011). Interhemispheric ERP asymmetries over inferior parietal cortex reveal differential visual working memory maintenance for fearful versus neutral facial identities. Psychophysiology, 48, 187-197.

Shackman, A. J., Maxwell, J. S., McMenamin, B. W., Greischar, L. L., \& Davidson, R. J. (2011). Stress potentiates early and attenuates late stages of visual processing. Journal of Neuroscience, 31, 11561161.

Shackman, A. J., Sarinopoulos, I., Maxwell, J. S., Pizzagalli, D. A., Lavric, A., \& Davidson, R. J. (2006). Anxiety selectively disrupts visuospatial working memory. Emotion, 6, 40-61.

Spielberger, C. D., Gorsuch, R. L., Lushene, R., Vagg, P. R., \& Jacobs, G. A. (1983). Manual for the State-Trait Anxiety Inventory. Palo Alto, CA: Consulting Psychologists Press.

Steppes, G., Luria, R., Fukuda, K., \& Gross, J. J. (2013). There's more to anxiety than meets the eye: Isolating threat-related attentional engagement and disengagement biases. Emotion, $13,520-528$.
Stout, D. M., \& Rokke, P. D. (2010). Components of working memory predict symptoms of distress. Cognition and Emotion, 24, 1293 1303.

Stout, D. M., Shackman, A. J., Johnson, J. S., \& Larson, C. L. (2015). Worry is associated with impaired gating of threat from working memory. Emotion, 15, 6-11.

Stout, D. M., Shackman, A. J., \& Larson, C. L. (2013). Failure to filter: Anxious individuals show inefficient gating of threat from working memory. Frontiers in Human Neuroscience, 7, 58.

Tottenham, N., Tanaka, J. W., Leon, A. C., McCarry, T., Nurse, M., Hare, T. A., Marcus, D. J., Westerlund, A., Casey, B. J., \& Nelson, C. (2009). The NimStim set of facial expressions: Judgments from untrained research participants. Psychiatry Research, 168, 242-249.

Weymar, M., Keil, A., \& Hamm, A. O. (2014). Timing the fearful brain: Unspecific hypervigilance and spatial attention in early visual perception. Social Cognitive and Affective Neuroscience, 9, 723-729.

Yiend, J. (2010). The effects of emotion on attention: A review of attentional processing of emotional information. Cognition and Emotion, 24, 3-47. 